\title{
Laboratory diagnosis of the rare anaemias: external quality assessment benefits patient care
}

\author{
Barbara De La Salle, ${ }^{1}$ Andrea Mosca, ${ }^{2}$ Renata Paleari, ${ }^{2}$ Vasileios Rapanakis, ${ }^{1}$ Keith Hyde ${ }^{1}$ \\ ${ }^{1}$ UK NEQAS for General Haematology, Watford, UK; ${ }^{2}$ University of Milan, Milan, Italy
}

Abstract

Since its introduction in the 1960 s, external quality assessment has developed to become an essential component of the quality management system of the diagnostic laboratory. External quality assessment provides a long term, retrospective view of laboratory performance, demonstrating the competence of the laboratory to others. The ENERCA project (the European Network for Rare and Congenital Anaemias) has established a list of core laboratory tests that are used in the diagnosis of rare and congenital anaemias, which has been used as the basis for questionnaires to laboratories, to establish the use and quality assurance of diagnostic testing in the congenital and rare anaemias, and to European EQA providers for services in this key area. In general, the provision of EQA for rare and congenital anaemias is widely variable with little provision for the very rare disorders. For the more common congenital anaemias, such as the haemoglobinopathies and thalassaemias, provision is better but there is variation in aspects of the scheme design, especially the frequency of distribution. Where laboratories did not take part in EQA for individual tests, or there was no EQA available, a desire to participate was expressed in $66 \%$ $(102 / 154)$ of cases. The provision of external quality assessment (EQA) services for rare disorders is a challenge. For many of these conditions, the number of patients in any one member state is very small with only a few laboratories providing diagnostic testing. In these cases, the development of pan-European or cross-border EQA may be the only means by which standardisation of methods and results can be achieved. An EQA survey of 243 laboratories for performance in $\mathrm{Hb}$ A2 quantification showed encouraging results in that there was a clear differentiation in the results from a beta Thalassaemia carrier and an individual with no evidence of Thalassaemia; however, a bias was observed between different methods of measurement.

\footnotetext{
Correspondence: Barbara De La Salle

E-mail: barbara.delasalle@whht.nhs.uk

CC Copyright B. De La Salle et al., 2013

Licensee PAGEPress, Italy

Thalassemia Reports 2013; 3(s1):e31

doi:10.4081/thal.2013.s1.e31
}

This article is distributed under the terms of the Creative Commons Attribution Noncommercial License (by-nc 3.0) which permits any noncommercial use, distribution, and reproduction in any medium, provided the original author(s) and source are credited.

Parts of this work were presented at the

"3rd Pan-European Conference on Haemoglobinopathies and Rare Anaemias", Limassol (Cyprus), 24-26 October 2012.

\section{Introduction}

The laboratory is central to the correct diagnosis of the patient with a rare or congenital anaemia. This central role becomes even more important when the screening for the carrier, who shows no symptoms of the condition, is considered. Patients, their relatives and carriers of the genes for rare and congenital anaemias have the reasonable expectation that their blood samples will be analysed appropriately, using effective methodology, and the results interpreted and reported correctly, in a timely manner.

Laboratory quality can be demonstrated through adherence to a quality management system, independently audited against professional guidelines, national and international standards. For diagnostic laboratories, there are a number of relevant international standards and other publications applicable to accreditation, for example, ISO 15189, ISO/IEC 17025, ISO 9001, ISO 9000 and publications by the European Communities Confederation of Clinical Chemistry $(1,2)$.

The stimulus for inter-laboratory comparability surveys in haematology were studies demonstrating poor performance in haemoglobin estimation, which led to the development of the ICSH reference haemoglobin standard and the introduction of Haematology external quality assessment in Britain in 1968 by the British Committee for Standardisation in Haematology. The development of the UK National External Quality Assessment Scheme in General Haematology (UKNEQAS(H)) followed the publication of results in 1969 from trials by Mitchell Lewis (3). Since that time, external quality assessment (EQA) has been widely adopted as a recognised part of laboratory quality management and participation in EQA is an essential requirement for laboratory accreditation. EQA complements but does not replace internal quality control (IQC). Whereas IQC will demonstrate that the results of one batch of investigations is comparable to the previous, EQA provides a long term, retrospective snapshot of a laboratory's performance in comparison with that of its peers.

Externally demonstrable laboratory competence, using external quality assessment and laboratory accreditation, is of paramount importance in the field of the rare and congenital anaemias. The World Health Organisation (WHO) defines a rare anaemia as one with fewer than 5 cases per 10,000 of population, and on a pan-European basis, this definition will cover the haemoglobinopathies and thalassaemias, as well as the much rarer disorders of the red cell enzymes, red cell membranes and erythropoiesis. The relative rarity of the disorders means that the frequency of the diagnostic test will be low and the number of centres undertaking the testing relatively few in any one country, often necessitating referral of the patient to a reference centre. In some cases this may require cross border referral. The European Network for Rare and Congenital Anaemias (ENERCA) initiative was founded to make this networking of expert centres a reality for patients, their relatives and professionals working with the rare anaemias.

As well as offering a measure of confidence in the performance of 
the individual laboratory (or even the individual clinician), EQA also allows some examination of the state of the art in laboratory methodology and the sharing of best practice through education. However, the low prevalence of rare anaemias makes the provision of EQA for the specialist laboratory tests a challenge for national EQA providers in individual member states since good EQA requires a minimum number of participating laboratories for financial and statistical viability.

Accordingly, the starting point for the work undertaken in the ENERCA workpackage on the Quality of Patient Care was to identify the list of core laboratory procedures that are consider essential for the diagnosis of rare anaemias, to assess the use of these core laboratory procedures across European laboratories, using a questionnaire (ENQUE$\mathrm{H1}$ ), to assess the provision of EQA for the core Laboratory procedures, using a questionnaire (ENQUE-H2) and to undertake an EQA exercise for $\mathrm{Hb} \mathrm{A} 2$.

\section{Methods}

\section{Core list of laboratory tests}

Using widely available laboratory manuals and text books, a core list of procedures applicable to the laboratory diagnosis of rare anaemias was prepared. The rare anaemias considered were those listed on the ENERCA website, classified into haemoglobin disorders, red cell enzyme disorders, red cell membrane disorders and paroxysmal nocturnal haemoglobinuria. In addition to specialist investigations, a section of general laboratory tests, such as complete (full) blood count (CBC or FBC), peripheral blood morphology and routine blood chemistry, was included. Conditions that rely on general laboratory investigations only, together with non-laboratory processes, such as clinical examination and imaging, were excluded.

\section{Questionnaire ENQUE-H1}

Questionnaire ENQUE-H1 was designed to establish the extent to which the different tests from the core list are used, their relevance to diagnosis and the quality assurance procedures utilised. The questionnaire was delivered electronically to the ENERCA network of centres and other centres across Europe.

\section{Questionnaire ENQUE-H2}

Questionnaire ENQUE-H2 was distributed to 31 European EQA provider organisations via the European Quality Assessment in Laboratory Medicine (EQALM) organisation, to establish the EQA provision for the investigations in the core list and the willingness of EQA provider organisations to collaborate across national boundaries.

The questionnaire sought information on the provision of EQA services for each test on the core list, including the analytes offered, frequency of distribution, type of survey material, number of participants, performance monitoring, wishlist of new provision, potential for collaboration and accreditation status.

\section{Hb A2 External Quality Assessment exercise}

Two specimen pools of human whole blood, with the unique identifiers 1004AH1 and 1004AH2, were collected into CPD - A1 anticoagulant, to which antibiotics were added. Specimen 1004AH1 was a single donation of blood from a beta thalassaemia carrier; specimen 1004 AH2 was a single donation of blood from an individual with no evidence of beta thalassaemia. Both specimen pools were prepared according to UK National External Assessment Scheme for General Haematology (UK NEQAS (H)) standard protocols.

Participants were recruited to this survey either via UK NEQAS (H) or the ENERCA organisation, each identified by a unique participant reference number. The specimen packages were distributed by first class post within the UK and by courier delivery outside the UK. Participants were asked to analyse both specimens by their regular method for $\mathrm{Hb} \mathrm{A} 2$ quantification and return their results (on-line or by fax) for analysis.

\section{Results}

\section{Core list of laboratory tests}

This list is lodged with ENERCA and is referenced in the ENERCA White Book (in preparation).

\section{Questionnaire ENQUE-H1}

The number of laboratories that reviewed or received the request to review the questionnaire is unknown because of the route of dissemination. A total of 70 responses were received from the following countries: Belgium, Czech Republic, Republic of Ireland, Italy, Luxembourg, The Netherlands, Spain, United Kingdom, Malta and Sweden

Thirty-five laboratories returned responses for laboratory investigations in the enzymopathies. The most commonly performed enzymopathy investigations were the quantitative assay for glucose-6-phosphate dehydrogenase (G6PD) activity (24/35 centres), the fluorescent spot test for G6PD activity (20/35 centres) and the quantitative assay for pyruvate kinase (PK) activity (8/35 centres). Most centres performed these investigations at least on a monthly basis. The quantitative assays of enzyme activity and molecular diagnosis were classed as the most relevant investigations: $63-75 \%$ classed quantitative assays as very relevant and $100 \%(2 / 2)$ molecular diagnosis. No investigation was classed as not relevant

Forty-nine laboratories returned responses for laboratory investigations in the haemoglobin disorders. The most commonly performed haemoglobinopathy investigations were those used for the screening, identification and quantification of haemoglobin fractions (42/49 providing HPLC testing, 26/49 $\mathrm{Hb}$ electrophoresis and 31/49 the sickle solubility test). Most centres performed these investigations at least on a daily or weekly basis. The more esoteric tests used to confirm the presence of, for example, $\mathrm{Hb} \mathrm{H}$ or altered oxygen affinity haemoglobins were performed only occasionally in most centres. Tests that allow the identification and quantification of haemoglobin fractions, such as HPLC and capillary electrophoresis were regarded as the most relevant, with $80-100 \%$ of the 49 respondents marking these as very relevant. Although very few respondents marked any test as not relevant, the less frequently performed tests, such as $\mathrm{Hb} \mathrm{H}$ bodies, Heinz bodies and unstable haemoglobins were more likely to be classified as only partially relevant.

Fifteen laboratories returned responses for laboratory investigations in the membrane disorders. The number of centres and the frequency of these tests are much lower than for the enzymopathies and haemoglobin disorders. The only tests provided by more than one centre are the EMA binding test (7/15 centres), the osmotic fragility test (7/15 centres) and the Pink test (3/15 centres). The majority of those undertaking the EMA binding test classified it as very relevant for diagnosis, in contrast to the osmotic fragility test, which was classified as partially relevant by all those that did it.

Thirty-one laboratories returned responses for tests from the general laboratory tests list. These tests, which include routine Blood Sciences investigations, are generally performed on a daily basis, as would be expected of tests that are used in the routine monitoring and diagnosis of many conditions. The most commonly performed general tests used in the diagnosis of rare anaemias are peripheral blood and bone marrow morphology (29/31 and 23/31 centres respectively) and 
the blood count (23/31 centres). Only liver iron is done infrequently, with a single centre performing the test on a monthly basis. Of the general laboratory tests, the blood count, together with peripheral blood and bone marrow morphology were the tests regarded as most relevant to diagnosis.

Very few tests were marked as not relevant for diagnosis in any category. There were 154 instances in which a centre did not take part or did not have EQA available for an individual test. In 102 (66\%) of these cases, the centre expressed the desire to participate in EQA.

\section{Questionnaire ENQUE-H2}

The questionnaire was distributed to $31 \mathrm{EQA}$ provider organisations within EQALM and responses were received from 16 (52\%). This included a supplier from Canada. (Table 1)

The provision of EQA for tests from the General section of the Core Tests list was good amongst the EQA providers that responded to the questionnaire, with 17/22 (77\%) of tests covered (see Figure 1). Five tests not covered by the EQA organisations that responded were: urine ferroxamine iron, serum transferrin receptor, liver iron, myocardial iron and zinc protoporphyrin.

Only 4 of the 16 EQA organisations provided EQA services for diagnostic tests associated with the haemoglobin disorders, and just 3 of these provided their services outside their own countries. The tests covered included sickle solubility; haemoglobin $(\mathrm{Hb})$ variant identification; quantification of $\mathrm{Hb} \mathrm{A} 2, \mathrm{Hb} \mathrm{F}$ and $\mathrm{Hb} \mathrm{S}$; $\mathrm{Hb} \mathrm{H}$ bodies; newborn sickle screening and molecular haemoglobinopathies (See Figure 2). EQA services were not available for unstable haemoglobins, Heinz bodies, oxygen affinity (p50) or globin chain synthesis among the organisations that responded.

There was little provision of EQA for the specialist diagnostic tests of other rare anaemias by the responding organisations. Of the core list of laboratory tests for red cell enzymopathies, red cell membrane disorders and paroxysmal nocturnal haemoglobinuria (PNH), EQA is only provided amongst the responders to the questionnaire for glucose-6phosphate dehydrogenase (G6PD) activity, Hb F by flow cytometry, Kleihauer (acid elution) slides, methaemoglobin and PNH by flow cytometry. In all cases, there was a single EQA provider for each test.
Service provision across national boundaries was available from $10 / 16$ (60\%) of EQA service providers. In addition, 11/15 (73\%) that responded would be prepared to offer new specialist EQA services in collaboration with other EQA providers.

There was a large variation in the frequency and number of specimens provided by different EQA organisations. For full blood count (FBC or CBC), for example, the number of specimens varied from 1 to 26 annually. A similar variation is seen for bilirubin (from 'as requested' to 52 specimens annually) and for $\mathrm{Hb} \mathrm{A} 2$ quantification (1 to 18 specimens annually).

The EQA providers were asked which tests they thought would most benefit from the development of new EQA services. Only 4 of the tests

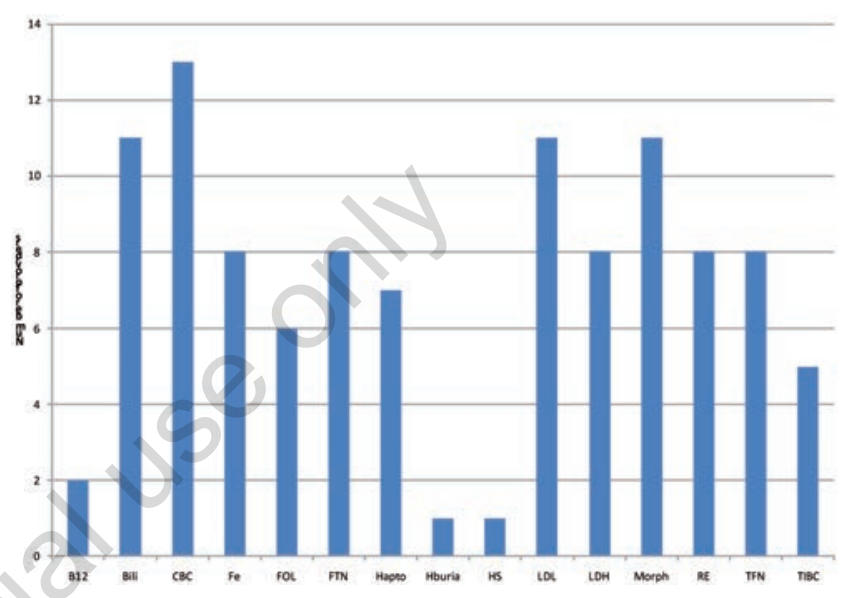

B12: vitamin B12 assay; Bili: bilirubin; CBC: complete blood count; Fe: serum iron; FOL: serum \&/or red cell folate; FTN: serum ferritin; Hapto: haptoglobin; Hburia: Haemoglobinuria; HS: urinary haemosiderin and bone marrow iron stain; LDL: low density lipoprotein; LDH: lactate dehydrogenase; Morph: periphera blood/bone marrow morphology; RE: reticulocyte count; TFN: transferrin; TIBC: total iron binding capacity.

Figure 1. EQA provision for tests from the General section of the core list of laboratory tests.
Table 1. Responses received to questionnaire ENQUE-H2

\begin{tabular}{lc} 
Country & EQA provider organisation \\
Canada & QMP-LS \\
Croatia & $\begin{array}{c}\text { Croatian Society of Medical Biochemists - } \\
\text { Committee for External Quality Control }\end{array}$ \\
\hline Czech Republic & SEKK \\
Denmark & DEKS \\
\hline France & AFSSAPS \\
& CTCB \\
Ireland & Irish EQAS \\
& RIQAS \\
\hline Norway & NOKLUS \\
Romania & RoEQALM \\
\hline Russia & SNEQAS \\
Slovenia & National Centre for EQA in Laboratory Medicine \\
\hline Spain & Sociedad Espanola de Hematologia y Hematorapia \\
Sweden & CSUALIS \\
\hline Switzerland & UK NEQAS \\
United Kingdom &
\end{tabular}

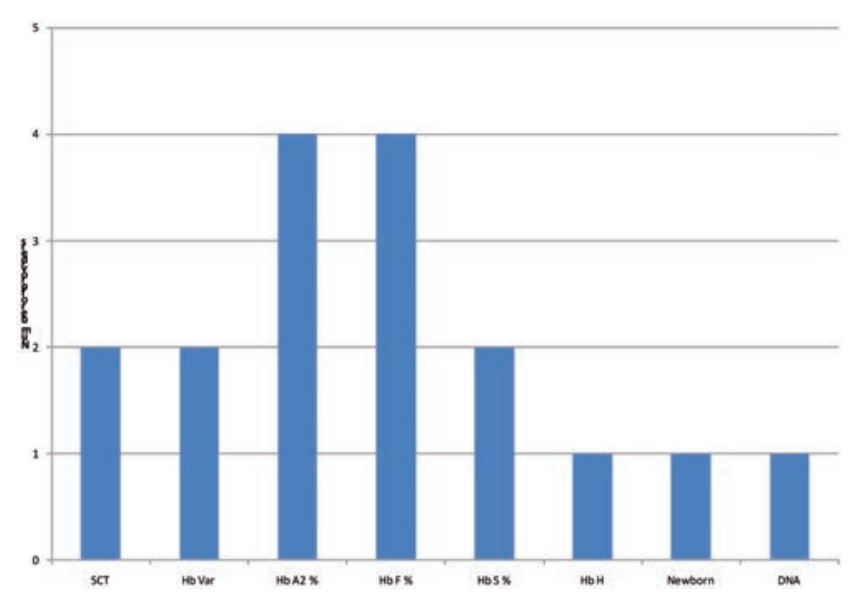

SCT: sickle screening test; $\mathrm{Hb}$ Var: Hb variant identification; $\mathrm{Hb}$ A2\%: quantification of $\mathrm{Hb} \mathrm{A} 2$; $\mathrm{Hb}$ F\%: quantification of $\mathrm{Hb} \mathrm{F} ; \mathrm{Hb} \mathrm{S} \%$ : quantification of $\mathrm{Hb} \mathrm{S} ; \mathrm{Hb} \mathrm{H}$ : $\mathrm{Hb} \mathrm{H}$ inclusion bodies; NB sickle: newborn sickle screening; DNA: molecular haemoglobinopathies.

Figure 2. EQA provision for tests for the diagnosis of haemoglobin disorders. 
listed did not have EQA provision by an alternative organisation within EQALM: unstable haemoglobins, Heinz bodies, serum transferrin receptor and pyruvate kinase (PK) activity.

The EQA providers reported the availability of a higher order reference method in less than $5 \%$ of analytes tested and this was not consistent between providers.

In nearly all cases, target values were derived from a consensus of participants' results. For tests such as morphology, a consensus of expert laboratories was also used. Over $90 \%$ of tests were subject to performance assessment. It was encouraging to note that $7 / 16$ of the EQA providers offered accredited services.

\section{$\mathrm{Hb}$ A2 EQA exercise}

The survey was distributed to 257 laboratories and 243 returned results (95\%). Table 2 shows the participants by country, together with the method principle used. The median \pm 2 estimated standard deviations (SD) for specimen 1004AH1 was $4.7 \pm 0.74 \%$ and for $1004 \mathrm{AH} 2$ $2.3 \pm 0.3 \%$. The range for each specimen was $3.3-6.4 \%$ for $1004 \mathrm{AH} 1$ and $1.6-2.9 \%$ for specimen 1004AH2. Table 3 shows the bias and percent bias of each instrument type against the consensus median for each specimen.

\section{Discussion}

The provision of EQA for general or routine diagnostic tests utilised in the diagnosis of rare anaemias is adequate amongst the EQA provider organisations within EQALM. These tests are used in the diagnosis and monitoring of a greater range of disorders than the rare anaemias, are widely available and hence have good EQA provision. The provision of EQA for more specialist tests however is not as good. Although responses to this questionnaire were received from a limited number of EQA providers, it included several large providers with a comprehensive range of services and also reflects the provision listed in other catalogues, such as that provided by the College of American Pathologists (www.cap.org) or the Centre for Disease Control (www.cdc.org).

Table 2. Participants and methods used for the Hb A2 EQA exercise, by country

\begin{tabular}{|c|c|c|c|c|c|}
\hline Country & HPLC & $\mathrm{CE}$ & $\mathrm{CC}$ & ELE & Total \\
\hline Cyprus & 1 & 1 & & & 1 \\
\hline Czech Republic & & 1 & & & 1 \\
\hline Denmark & 2 & & & & 2 \\
\hline France & 1 & & & & 1 \\
\hline Greece & 2 & & & & 2 \\
\hline Ireland & 7 & & & & 7 \\
\hline Italy & 20 & 1 & & & 21 \\
\hline Luxembourg & 1 & 1 & & & 2 \\
\hline Norway & 1 & 1 & & & 2 \\
\hline Portugal & 19 & & 2 & 1 & 22 \\
\hline Spain & 1 & & & & 1 \\
\hline Sweden & 2 & & & & 2 \\
\hline Switzerland & 2 & & & & 2 \\
\hline UK & 168 & & 4 & & 172 \\
\hline TOTAL & 231 & 5 & 6 & 1 & 243 \\
\hline
\end{tabular}

$\mathrm{CE}=$ Capillary Electrophoresis; $\mathrm{CC}=$ column chromatography; ELE=Electrophoresis and elution; HPLC=High Performance Liquid Chromatography.
The relatively poor provision of EQA for RA diagnostic tests reinforces the need for collaborative operation where possible. For the laboratory, the choice of EQA programme is made difficult by the differences in the service offered between providers, for example the very wide variation in the frequency of provision. The responsibility for the selection of an EQA scheme appropriate to the laboratory's needs lies with the laboratory and this requires a diversity of EQA provision; however, patients have the right to expect that the EQA services conform to a recognised quality standard and that the scope of the EQA programme is clear. For this reason, EQA providers should work to improve their services through accreditation to international standards, such as IS017043. This is particularly important if EQA services are provided across national boundaries.

Of the diagnostic tests for which EQA is not available, ENERCA has identified pyruvate kinase (PK) activity as the procedure for which it is most feasible to develop a pilot EQA scheme. Relatively few centres may provide qualitative and/or quantitative $\mathrm{PK}$ assay within any one EU member state and the interpretation of the results is challenging, meaning that an effective EQA programme would have an impact. The most effective model would be to develop a Europe wide EQA programme, using the expertise of a consortium of experts in EQA provision, laboratory diagnosis and clinical management of PK deficiency.

The provision of high quality EQA, coupled with educational support, is an important component of a quality management system by which laboratory performance can be improved and maintained, and thus supports the development of laboratory services for patients with rare anaemias. The majority of the EQA providers indicated a willingness to provide their services across national borders or to collaborate in the development of new, specialist services. This is important where the EQA programme may only be statistically viable if participants are recruited from a number of countries. However, the availability of sufficient volumes of stable survey material, funding models that restrict the provision of EQA to a single country, the cost of transportation, restrictive customs rules, language and differences in local medical practice all pose barriers to cross border service delivery.

International accreditation standards and support from professional bodies with an international profile, such as ENERCA, the International Federation of Clinical Chemistry (IFCC), the International Council for Standardisation of Haematology (ICSH) and the World Health Organisation (WHO), will be of assistance to overcome the barriers to the cross-border provision of EQA for RA.

The results returned for the $\mathrm{Hb} \mathrm{A} 2 \mathrm{EQA}$ exercise were encouraging, in that the $95 \%$ confidence limits for specimen 1004AH1 were 3.96 $5.44 \%$, which is clearly raised and consistent with a diagnosis of beta thalassaemia trait. The $95 \%$ confidence limits for $1004 \mathrm{AH} 2$ were 2.00 $2.60 \%$, which is well within the accepted range for a patient with no evidence of beta thalassaemia trait. Within the UK, the accepted cut-off $\mathrm{Hb} \mathrm{A} 2$ value for the diagnosis of beta thalassaemia trait is $3.5 \%$ or above (4). The reported range of results for specimen 1004AH1 was 3.3 - $6.4 \%$; 2 laboratories reported a value of less than $3.5 \%$ for this specimen, below the UK cut-off value for the diagnosis of beta thalassaemia trait. The reported range for specimen 1004AH2 was $1.6-2.9 \%$ and no laboratory reported a raised $\mathrm{Hb} \mathrm{A} 2$ value for this specimen.

Although many of the laboratories that took part in the $\mathrm{Hb}$ A2 EQA exercise were within the United Kingdom, recruited as part of the UK NEQAS participant base, results were returned from a total of 15 European countries. The majority of participants (231/243) used High Performance Liquid Chromatography (HPLC), with the most commonly used HPLC instruments being the BioRad Laboratories Variant 2 analyser (76/243, with either the beta thalassaemia short or the dual kit), the Menarini HA8160 (54/243) and the Tosoh G7 and G8 analysers (50/243).

The different methods used by participants displayed method related bias against the consensus median result for each specimen. Although 
Table 3. Method bias seen in the Hb A2 EQA survey, by method principle / analyser used.

\begin{tabular}{|c|c|c|c|c|c|c|c|}
\hline \multirow[t]{2}{*}{ Hb A2 METHOD } & \multirow[t]{2}{*}{ No. } & \multicolumn{3}{|c|}{ 1004AH1 } & \multicolumn{3}{|c|}{ 1004AH2 } \\
\hline & & Bias & Bias \% & Range \% & Bias & Bias \% & Range \% \\
\hline Sebia Capillaris & 5 & 0.10 & $2.13 \%$ & $-2.1 \%$ to $+6.4 \%$ & -0.08 & $-3.48 \%$ & $-8.7 \%$ to $0 \%$ \\
\hline Column Chromatography & 6 & 0.02 & $0.35 \%$ & $-6.4 \%$ to $+12.8 \%$ & 0.15 & $6.52 \%$ & $-4.4 \%$ to $+26.1 \%$ \\
\hline Electrophoresis & 1 & -1.40 & $-29.79 \%$ & $\sim$ & -0.50 & $-21.74 \%$ & $\sim$ \\
\hline $\begin{array}{l}\text { HPLC: } \\
\text { Method not stated } \\
\text { Beckman Coulter Gold }\end{array}$ & $\begin{array}{l}4 \\
1\end{array}$ & $\begin{array}{c}0.30 \\
-0.10\end{array}$ & $\begin{array}{l}6.38 \% \\
-2.13 \%\end{array}$ & $\begin{array}{c}-2.1 \% \text { to }+12.8 \% \\
\sim\end{array}$ & $\begin{array}{c}0.08 \\
-0.30\end{array}$ & $\begin{array}{c}3.26 \% \\
-13.04 \%\end{array}$ & $\begin{array}{c}-8.7 \% \text { to }+21.7 \% \\
\sim\end{array}$ \\
\hline $\begin{array}{l}\text { BioRad D10, Dual Program Kit } \\
\text { BioRad Variant Classic }\end{array}$ & $\begin{array}{l}21 \\
12\end{array}$ & $\begin{array}{l}0.25 \\
-0.07\end{array}$ & $\begin{array}{l}5.37 \% \\
-1.42 \%\end{array}$ & $\begin{array}{l}-8.5 \% \text { to } 25.5 \% \\
-6.4 \% \text { to }+4.3 \%\end{array}$ & $\begin{array}{l}0.06 \\
0.07\end{array}$ & $\begin{array}{l}2.48 \% \\
2.90 \%\end{array}$ & $\begin{array}{l}-13.0 \% \text { to }+26.1 \% \\
-4.4 \% \text { to }+17.4 \%\end{array}$ \\
\hline $\begin{array}{l}\text { BioRad Variant II; Beta-thal } \\
\text { BioRad Variant II; Dual Kit }\end{array}$ & $\begin{array}{l}51 \\
25\end{array}$ & $\begin{array}{c}-0.01 \\
0.09\end{array}$ & $\begin{array}{l}-0.29 \% \\
1.87 \%\end{array}$ & $\begin{array}{l}-14.9 \% \text { to }+14.6 \% \\
-4.3 \% \text { to }+10.6 \%\end{array}$ & $\begin{array}{r}0.06 \\
-0.09\end{array}$ & $\begin{array}{r}2.47 \% \\
-3.83 \%\end{array}$ & $\begin{array}{l}-13.0 \% \text { to }+17.4 \% \\
-17.4 \% \text { to }+4.4 \%\end{array}$ \\
\hline Menarini HA8160 & 54 & -0.40 & $-8.43 \%$ & $-29.8 \%$ to $+6.4 \%$ & 0.04 & $1.61 \%$ & $-30.4 \%$ to $+21.7 \%$ \\
\hline Menarini Hb Gold & 5 & -0.40 & $-8.51 \%$ & $-14.9 \%$ to $0 \%$ & -0.08 & $-3.48 \%$ & $-8.7 \%$ to $0 \%$ \\
\hline Primus Ultra 2 & 6 & -0.05 & $-1.06 \%$ & $-2.1 \%$ to $0 \%$ & -0.25 & $-10.87 \%$ & $-13.0 \%$ to $-8.7 \%$ \\
\hline Shimadzu LC10 & 1 & -0.60 & $-12.77 \%$ & $\sim$ & 0.00 & $0.00 \%$ & $\sim$ \\
\hline Tosoh G7 & 37 & 0.82 & $17.42 \%$ & $+4.3 \%$ to $+36.2 \%$ & -0.04 & $-1.53 \%$ & $-17.4 \%$ to $+13.0 \%$ \\
\hline Tosoh G8 & 13 & 0.52 & $11.13 \%$ & $+2.1 \%$ to $+23.4 \%$ & -0.11 & $-4.68 \%$ & $-13.0 \%$ to $+8.7 \%$ \\
\hline Varian Prostar & 1 & 0.10 & $2.13 \%$ & $\sim$ & -0.30 & $-13.04 \%$ & $\sim$ \\
\hline ALL METHODS & -0.05 & $-1.10 \%$ & & -0.08 & $-3.53 \%$ & & \\
\hline
\end{tabular}

Optimum $=$ Bias $<2.5 \%$; Minimum $=$ Bias $<7.6>5.1 \%$; Desirable $=$ Bias $<5.1>2.5 \%$; Bias $>7.6 \%$.

there are no consensus limits for the analytical quality for $\mathrm{Hb} \mathrm{A} 2$ measurement, minimum, desirable and optimum \%bias limits have been proposed $(5,6)$. When these are applied to the percent bias values obtained for raised $\mathrm{Hb} \mathrm{A} 2$ specimen 1004AH1, the Tosoh instruments showed a positive bias and the Menarini HA8160 instruments a negative bias (see Table 3). This observation fits with a recent analysis of historical Hb A2 data issued by UK NEQAS (H) (7) and with a comparison of current high-performance methods for $\mathrm{Hb} \mathrm{A} 2$ measurement by Paleari et al.(8). However, this observation was less evident with the normal specimen 1004AH2 and in fact none of the major HPLC methods showed a method bias outside the desirable range of less than $5.1 \%$. However, the bias shown by individual laboratories is much more variable.

The analysis of the method bias against a consensus median must be interpreted with caution. The consensus median is influenced in favour of the instrument with the greatest number in the analysis, which in this case is the BioRad instrument group, particularly the BioRad Variant 2 analyser. The numbers of Menarini HA8160 (54) and Tosoh G7/G8 instruments (50) are significant however and should be sufficient to make the analysis representative. It must be emphasized that these results indicate the relative bias between analysers and should not be interpreted as indicating that one instrument is more correct than another. In conclusion, the results of this EQA survey are encouraging but support the view that more work is needed on the calibration of instruments and harmonization of the reference intervals in use, for the benefit of patient care. The International Council for Standardisation of Haematology (ICSH) has recently issued an updated guideline on $\mathrm{Hb}$ A2 quantification and interpretation (9), encompassing current high throughput methods.

A networking organisation such as ENERCA has a key role in the facilitating collaborative working between experts in the development of EQA services and in publicising their availability to laboratory professionals.

\section{References}

1. European Communities Confederation of Clinical Chemistry: Essential Criteria for Quality Systems of Medical Laboratories; Eur J Clin Chem Clin Bioch (1997); 35: 121-132

2. European Communities Confederation of Clinical Chemistry: Additional Essential Criteria for Quality Systems of Medical Laboratories; Eur J Clin Chem Clin Bioch (1998); 36: 249-252

3. Lewis S.M. \& Burgess B.J. (1969). Quality Control in Haematology: Report of Interlaboratory Trials in Britain. British Journal of Haematology 4: 253-256.

4. NHS Sickle and Thalassaemia Screening Programme, Handbook for Laboratories 2nd Edition (2009), ISBN 13: 978-0-9554319-2-0, http://www.sct.screening.nhs.uk

5. Paleari R., Giambona A., Cannata M., Leto F., Maggio A. \& Mosca A. (2007). External quality assessment of hemoglobin A2 measurement: data from an Italian pilot study with fresh whole blood samples and commercial HPLC systems. Clin Chem Lab Med 45:88-92

6. Mosca A., Paleari R., Giambona A., Cannata M., Leto F., Li Muli R. \& Maggio A. (2007). Esperienze pilota sulla Valutazione Esterna di Qualità della misura dell'emoglobina A2 con campioni di sangue intero e metodiche HPLC commerciali. Biochimica clinica 31:105-110

7. Batterbee H., De la Salle B., McTaggart P., Doré C., Wild B. \& Hyde K. (2010). Evaluation of UK NEQAS (H) Hb A2 and related performance data; available at http//www.ukneqash.org (accessed 1 September 2012)

8. Paleari R., Gulbis B., Cotton F. \& Mosca A (2012). Interlaboratory comparison of current high-performance methods for $\mathrm{Hb} \mathrm{A} 2$. International Journal Laboratory Haematology. 34: 362-368.

9. Stephens A.D., Angastiniotis M., Baysal E., Chan V., Fucharoen S., Giordano P.C,. Hoyer J.D., Mosca A. \& Wild B. (2012), on behalf of the International Council for the Standardisation of Haematology. ICSH Recommendations for the measurement of $\mathrm{Hb} \mathrm{A} 2$. International Journal Laboratory Haematology. 34: 1-13. 